\title{
Study of the Myocardial Contraction and Relaxation Velocities through Doppler Tissue Imaging Echocardiography. A New Alternative in the Assessment of the Segmental Ventricular Function
}

\author{
Carlos Eduardo Suaide Silva, Luiz Darcy Cortez Ferreira, Luciana Braz Peixoto, \\ Claudia Gianini Monaco, Manuel Adán Gil, Juarez Ortiz \\ São Paulo, SP - Brazil
}

\begin{abstract}
Objective-Dopplertissue imaging (DTI) enables the study of the velocity of contraction and relaxation of myocardial segments. We established standards for the peak velocity of the different myocardial segments of the left ventricle in systole and diastole, and correlated them with the electrocardiogram.
\end{abstract}

Methods - We studied 35 healthy individuals $(27$ were male) with ages ranging from 12 to 59 years $(32.9 \pm 10.6)$. Systolic and diastolic peak velocities were assessed by Doppler tissue imaging in 12 segments of the left ventricle, establishing their mean values and the temporal correlation with the cardiac cycle.

Results - The means (and standard deviation) of the peak velocities in the basal, medial, and apical regions (of the septal, anterior, lateral, and posterior left ventricle walls) were respectively, in $\mathrm{cm} / \mathrm{s}, 7.35(1.64), 5.26(1.88)$, and 3.33(1.58) in systole and 10.56(2.34), 7.92(2.37), and 3.98(1.64) in diastole. The mean time in which systolic peak velocity was recorded was $131.59 \mathrm{~ms}( \pm 19.12 \mathrm{~ms})$, and diastolic was $459.18 \mathrm{~ms}( \pm 18.13 \mathrm{~ms})$ based on the peak of the $R$ wave of the electrocardiogram.

Conclusion - In healthy individuals, maximum left ventricle segment velocities decreased from the bases to the ventricular apex, with certain proportionality between contraction and relaxation $(P<0.05)$. The use of Doppler tissue imaging may be very helpful in detecting early alterations in ventricular contraction and relaxation.

Keywords: Doppler tissue imaging, ventricular function, echocardiography

Noninvasive Heart Division - São Paulo

Mailing address: Carlos Eduardo Suaide Silva - Centro de Cardiologia Não Invasiva Rua Cubatão, 726 - 04013-002 - São Paulo, SP - E-mail: csuaide@ cardiol.br
The Doppler effect was described in 1842 by the Austrian physicist Christian Johann Doppler, but, its use in medicine, especially in echocardiography, did not begin until one hundred years later ${ }^{1}$.

Only after the 1980 s was Doppler echocardiography incorporated into routine diagnosis, significantly improving the method, through the access of valuable information about heart physiology, which was previously not available. The main contribution of this technique was to enable assessment of the direction, the sense, and the velocity in which the blood moves inside the heart and vessels and, therefore, making a significant contribution to the study of valvulopathies and congenital cardiopathies ${ }^{1}$. However, additional benefits concerning information about the assessment of global ventricular function and in the assessment of segmental function have not been widely discussed. Two-dimensional echocardiography enabled the study of the regional myocardial contractile function, and the performance of new procedures under physical or pharmacological stress, enhancing the sensitivity and specificity of the examinations of patients at rest. However, the interpretation of the segmental function in these tests can be limited by the learning curve, quality of imaging, and accuracy of the interpretation of the results ${ }^{2}$. Additionally, we cannot evaluate myocardial diastolic or relaxation function properly using only two-dimensional echocardiography. Tissue Doppler imaging, on the other hand, is a fairly new technique that allows assessing quantitatively, the velocity of myocardial movement, during systole and diastole ${ }^{3}$. The first studies of Doppler tissue imaging appeared in the late $1980 \mathrm{~s}^{4}$, but the method increased in importance with studies in Edinburgh led by Sutherland et al ${ }^{5}$ and Fleming et al ${ }^{6}$ in 1994. 
Basically, Doppler tissue imaging was developed based on the physical differences between moving blood and moving myocardium. The myocardium moves with substantially lower velocities ( 4 to $15 \mathrm{~cm} / \mathrm{s}$ versus 40 to $150 \mathrm{~cm} / \mathrm{s}$ of the blood flow) and produces signals with amplitudes hundred times greater than those of blood ${ }^{7,8}$. Conventional Doppler uses high-pass filtering to reject the "noises" of low velocity and high amplitude, originating at the ventricular wall and, thereby, allowing the calculation of blood flow velocity. The replacement of this filter for another enables the acquisition of the myocardial signals and the instant calculation of the velocity of movement at any specific point of the cardiac muscle. This technique has been used to quantify the velocity of segmental movement of the ventricular myocardium, as well as, to study regional systolic and diastolic function ${ }^{9}$.

The purpose of this study was to perform the analysis of the maximum velocity of contraction and relaxation of the several segments of the left ventricular myocardium in patients without cardiopathy, establishing their temporal correlation with the cardiac cycle.

\section{Methods}

Thirty-five healthy volunteers were prospectively

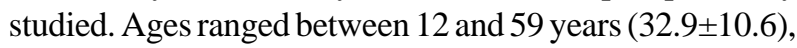
and 27 individuals were male. Individuals who had cardiac abnormalities on clinical, electrocardiographic, and echocardiographic examination were excluded. The studies were performed using commercially available pulsed Doppler systems GE-Vingmed model System V, with multifrequency transducers (1.5 a 3.6 MHz), and harmonics capitation and Doppler tissue imaging. After conventional transthoracic echocardiography, Doppler tissue imaging was performed using a 4- and 2-chamber view. We analyzed the basal, medial, and apical segments of the septal and lateral walls with a 4chamber view and the same segments in the anterior and inferior walls with a 2-chamber view, with a total of 12 segments studied in each case (fig. 1). The examinations were digitally stored (looping of a complete cardiac cycle) for later assessment of the peak velocity of contraction (peak systolic velocity) and relaxation (peak diastolic velocity) in each of the 12 segments. These velocities were obtained with a spectral tissue Doppler curve, placing the volume sample in each of the above-mentioned segments. We also determined elapsed time in milliseconds, from the $\mathrm{R}$ wave of the electrocardiogram in which these velocities occurred (fig. 2).

We calculated the mean peak systolic and diastolic velocities observed in the basal, medial, and apical regions of the lateral, septal, anterior, and inferior walls.

Statistical analysis was used to calculate the mean and standard deviation of all the variables studied. Comparison between the peak velocity (systolic and diastolic) observed in the different segments was performed with the Student's $t$ test using a 5\% significance level.

\section{Results}

Systolic and diastolic peak velocity means and standard deviation obtained from the 12 segments studied, as well as the mean velocity per region (basal, medial, and apical) are presented in table I.

The mean and standard deviation of the times in which maximal velocities of contraction and relaxation were recorded in relation to the peak of the $\mathrm{R}$ wave of the electrocardiogram are in table II.

The mean systolic and diastolic regional peak velocities (basal, medial, and apical) are in figure 2 .

We observed that the maximum motion velocities of the left ventricle myocardial segments decreased from the bases to the ventricular apex, with proportionality between contraction and relaxation. The mean peak velocities during relaxation were significantly higher $(P<0.05)$ than the mean peak systolic velocities, in a great number of the segments. That is, the maximum motion velocity of the left ventricular myocardium occurs during diastole, especially in the rapid
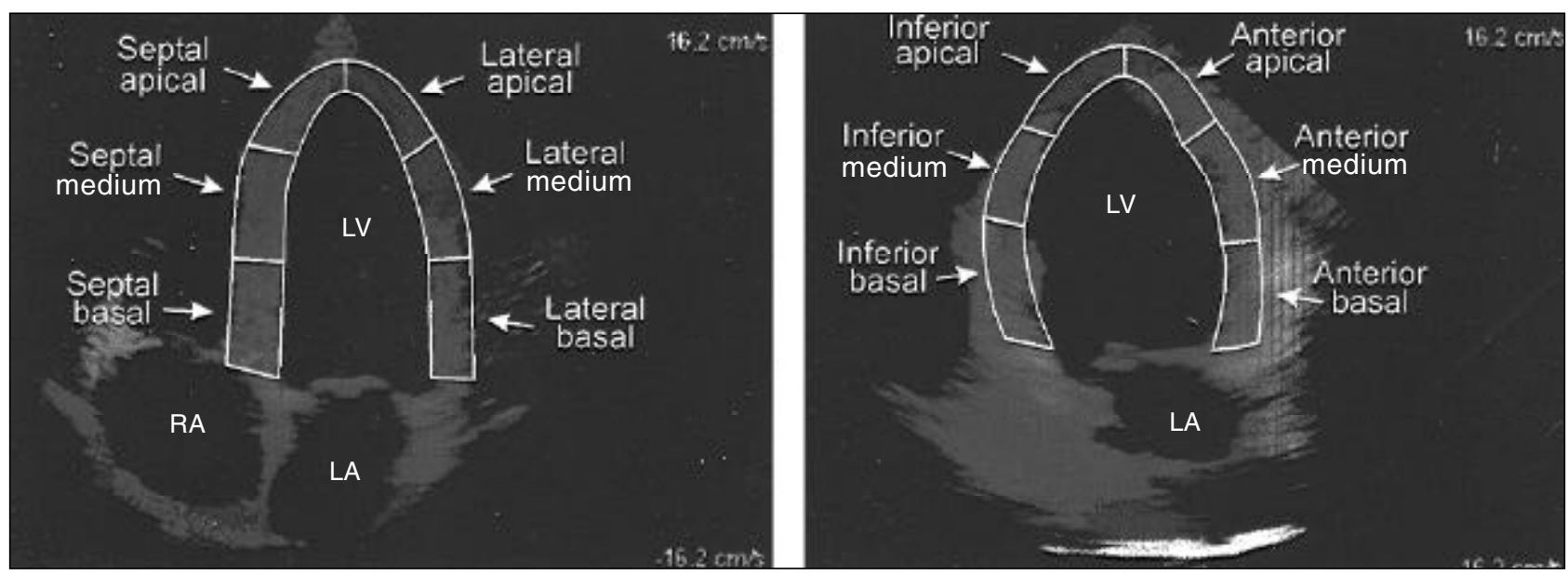

Fig. 1 - Left panel: a 4 -chamber view showing the segments assessed of the septal and lateral left ventricle (LV) wall. Right panel: a 2-chamber view showing the segments assessed of the inferior and anterior left ventricle walls. LA- left atrium; RA- right atrium. 


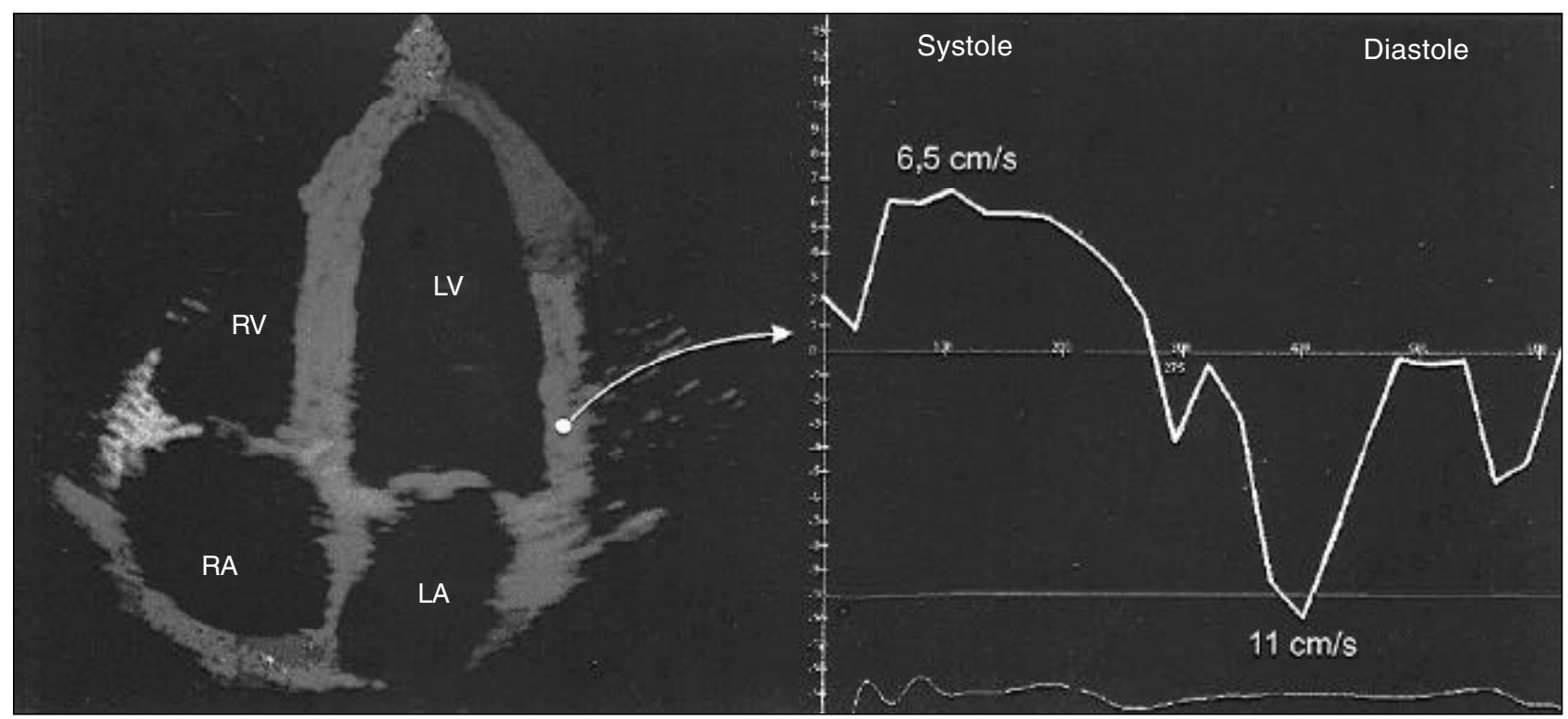

Fig. 2 - Left panel: a 4-chamber apical view in Doppler tissue imaging showing the lateral wall where the contraction and relaxation velocities are being measured. Right panel Digital profile of the velocity. The positive curve shows the peak systolic velocity $(6.5 \mathrm{~cm} / \mathrm{s})$ and the negative peak diastolic velocity $(11 \mathrm{~cm} / \mathrm{s})$. RA- right atrium; LA- left atrium; RV-right ventricle; LV- left ventricle.

\begin{tabular}{|c|c|c|c|c|c|}
\hline \multicolumn{6}{|c|}{$\begin{array}{l}\text { Table I - Mean of peak velocities, systolic and diastolic, by segment } \\
\text { (and region) and the respective standard deviation (sd) in } \mathrm{cm} / \mathrm{s}\end{array}$} \\
\hline \multirow{3}{*}{ Segment } & \multicolumn{2}{|c|}{$\begin{array}{l}\text { Apical } \\
4 \text { - chamber }\end{array}$} & \multicolumn{2}{|c|}{$\begin{array}{l}\text { Apical } \\
2 \text { - chamber }\end{array}$} & \multirow[t]{2}{*}{$\begin{array}{l}\text { Mean } \\
\text { by region }\end{array}$} \\
\hline & Lateral & Septal & Anterior & Inferior & \\
\hline & Sys. Dias. & Sys. Dias. & Sys. Dias. & Sys. Dias. & Sys. Dias. \\
\hline \multirow{6}{*}{$\begin{array}{l}\text { Apical } \\
\text { (dp) } \\
\text { Medium } \\
\text { (dp) } \\
\text { Basal } \\
\text { (dp) }\end{array}$} & 4.544 .12 & 2.593 .57 & 2.934 .20 & $2.97 \quad 3.99$ & $3.33 \quad 3.98$ \\
\hline & 1.931 .52 & $0.71 \quad 1.61$ & 1.432 .09 & $1.07 \quad 1.39$ & $1.58 \quad 1.64$ \\
\hline & 6.828 .63 & 4.367 .41 & 4.957 .36 & $4.93 \quad 8.05$ & 5.267 .92 \\
\hline & 2.442 .64 & $0.94 \quad 1.65$ & 1.972 .97 & $1.00 \quad 1.95$ & $\begin{array}{ll}1.88 & 2.37\end{array}$ \\
\hline & 8.0710 .99 & $6.53 \quad 9.65$ & 7.7310 .45 & $\begin{array}{ll}7.12 & 10.99\end{array}$ & 7.3510 .56 \\
\hline & 2.002 .77 & $1.05 \quad 1.52$ & 1.932 .83 & $0.93 \quad 1.91$ & $1.64 \quad 2.34$ \\
\hline
\end{tabular}

filling phase (fig. 2). Only in the lateral apical segment were similar values of motion velocities recorded, in systole compared with diastole $(P=0.242)$.

The mean time for reaching maximum contraction velocity (systolic peak velocity) was $131.59 \mathrm{~ms}( \pm 19.12 \mathrm{~ms})$ counting from the $\mathrm{R}$ wave of the electrocardiogram, whereas the peak diastolic velocity occurred, on average, at $459.18 \mathrm{~ms}$
$( \pm 18.13 \mathrm{~ms})$, after the same electrical phenomenon always during the rapid ventricular filling phase (E wave of the mitral inflow).

The mean of the peak systolic regional velocities was significantly greater in the basal segments, in relation to that in the medial region $(P<0.001)$, which, in turn, was significantly greater then that observed in the apical region $(P<0.001)$.

Analysis of the mean of the maximum motion velocities of the myocardium in relation to the phases of the cardiac cycle (fig. 4) demonstrates that these values were significantly greater in diastole than in systole in each region $(P<0.001)$.

\section{Discussion}

Although the ejection fraction and fractional shortening express the ventricular performance with confidence, suitable assessment of the myocardium in the different phases of the cardiac cycle may help to better understand some pathologic conditions. Alterations in myocardial motion during the ventricular rapid filling phase is consi-

\begin{tabular}{|c|c|c|c|c|c|c|c|c|}
\hline \multirow{3}{*}{ Segment } & \multicolumn{3}{|c|}{ Apical 4-chamber } & \multicolumn{5}{|c|}{ Apical 2-chamber } \\
\hline & \multicolumn{2}{|c|}{ Lateral } & \multicolumn{2}{|c|}{ Septal } & \multicolumn{2}{|c|}{ Anterior } & \multicolumn{2}{|c|}{ Inferior } \\
\hline & Systolic & Diastolic & Systolic & Diastolic & Systolic & Diastolic & Systolic & Diastolic \\
\hline Apical & 106 & 473 & 144 & 453 & 111.2 & 478 & 133 & 463 \\
\hline (dp) & 43.8 & 61.7 & 52.3 & 113 & 43.8 & 124 & 51.6 & 60.7 \\
\hline Medium & 88 & 480 & 146 & 474 & 118 & 478 & 137 & 496 \\
\hline$(\mathrm{dp})$ & 25.8 & 55.8 & 46.3 & 50.6 & 50.8 & 89.9 & 45.9 & 84.4 \\
\hline Basal & 103 & 466 & 124 & 457 & 110 & 438 & 125 & 466 \\
\hline (dp) & 31.2 & 66.6 & 39.8 & 72.3 & 44.1 & 88.2 & 37.6 & 84.1 \\
\hline
\end{tabular}




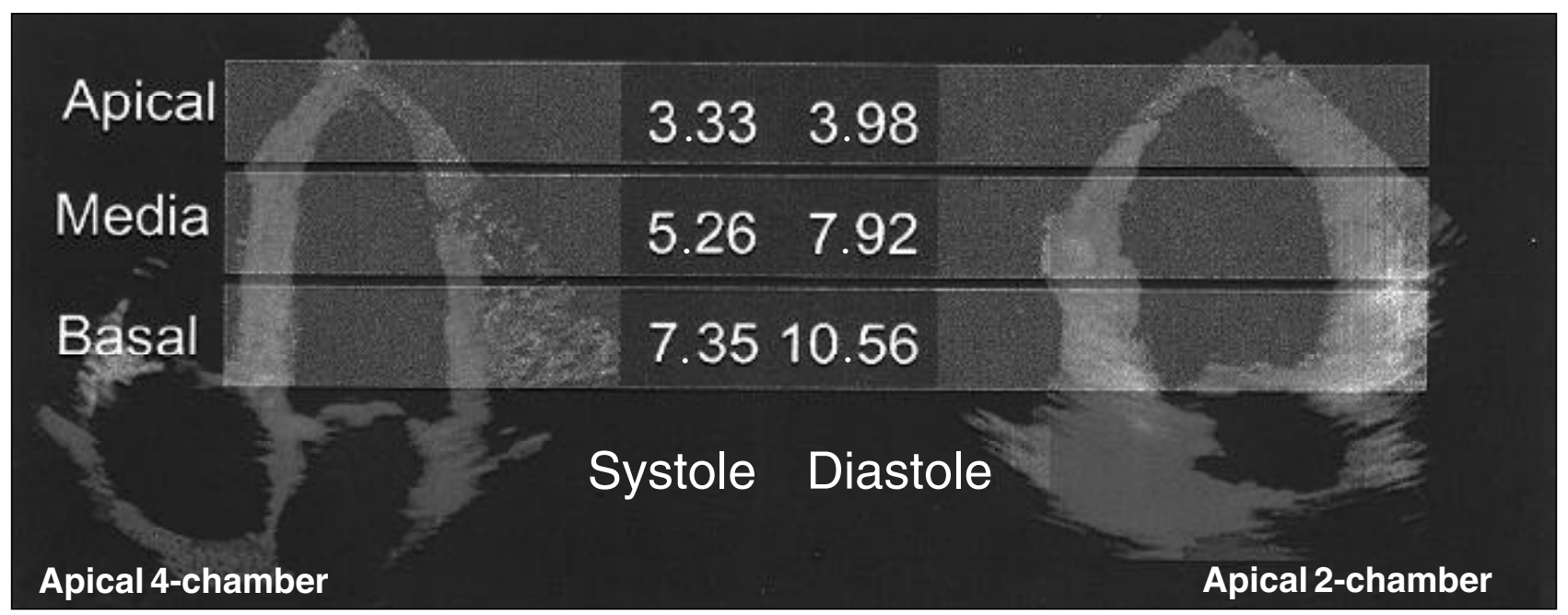

Fig. 3 - Means of peak systolic and diastolic velocities (in $\mathrm{cm} / \mathrm{s}$ ) of the medial and apical basal segments showing that the velocities measured decreased from the base to the ventricular apex and that the maximum movement velocity is greater during relaxation than contraction.

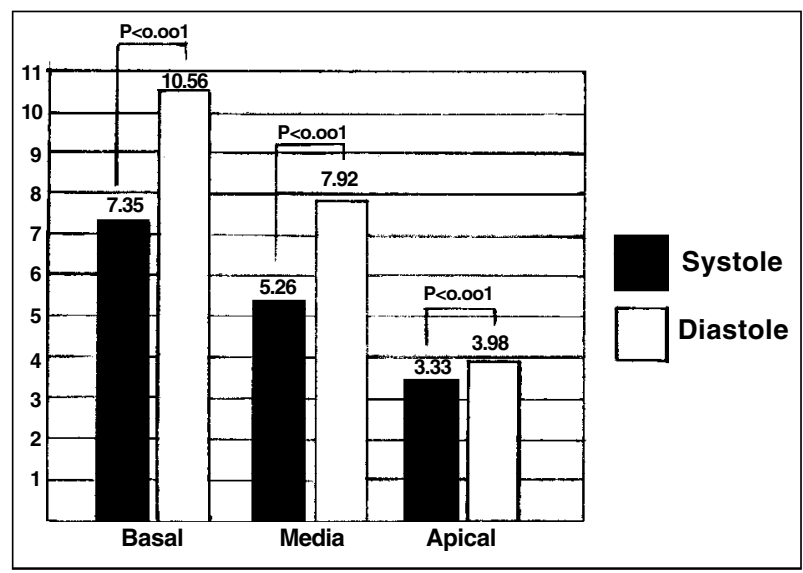

Fig. 4-Mean of systolic and diastolic peak velocities by region (basal, medial, and apical).

dered an early marker of cardiac commitment in hypertension ${ }^{10}$. Also, in the early stages of coronary disease, an increase may occur in the time of $E$ wave deceleration in the mitral inflow, even though systolic function is preserved. Doppler tissue imaging can also demonstrate: alterations in ventricular relaxation, initially, in certain myocardial segments, preceding alterations in the global commitment of ventricular filling. Regional diastolic dysfunction may be, therefore, a very early indicator of ischemia ${ }^{10}$, but to recognize parameters alterations obtained with Doppler tissue imaging, it is necessary to know its normal values ${ }^{11,12}$.

Our study assessed, in healthy individuals, the maximum velocities of contraction and relaxation of the basal, medial, and apical segments of the lateral, septal, anterior, and inferior left ventricle walls. In addition, we established the time in which these velocities occurred in relation to the electrocardiogram in the population studied.

We observed that the maximum velocities of contraction and relaxation decreased from the base to the ventricular apex and that the maximum velocities of relaxation are signi- ficantly greater than the velocities of contraction, in the majority of myocardial segments.

Despite this, a limitation of this method is that it does not enhance the difference between the velocities observed in the apical and basal segments. Doppler tissue imaging, as with any type of Doppler, depends on the angle at which the ultrasound beam reflects, that is, the more parallel the beam is to the direction of the main displacement of the structure to be analyzed, the more accurate is the velocity measured measurement ${ }^{13,14}$. The motion of the left ventricular myocardium is very complex. At least 3 types of fiber shortening occur, longitudinal, circumferential, and spiral, and the final motion results from these three. The resulting vector is generally directed to a site within the ventricular cavity, about $70 \%$ of the distance between the base and the apex (fig. 5). Therefore, the velocity vector of the mitral annulus region is that more parallel to the ultrasound beam considering the views we used (apical 4 and 2 chambers). In the apical region, the result of the motion is perpendicular to the ultrasound beam, significantly underestimating the velocity measured by Doppler tissue imaging. Thus, the measures performed in the apical region must be assessed carefully. They may be less accurate in absolute values in relation to those obtained in basal segments, but to compare the same individual in consecutive studies, either at rest or under stress, they can be used with acceptable confidence.

It is possible that this dependence on the angle explains the great amplitude of the standard deviation observed in the measures performed in the ventricular apical region.

Likewise, such restrictions in the velocity measures imposed by the angle of incidence of the ultrasound do not hinder the comparison between the velocities obtained in systole and diastole, because no angle variation occurs, in 


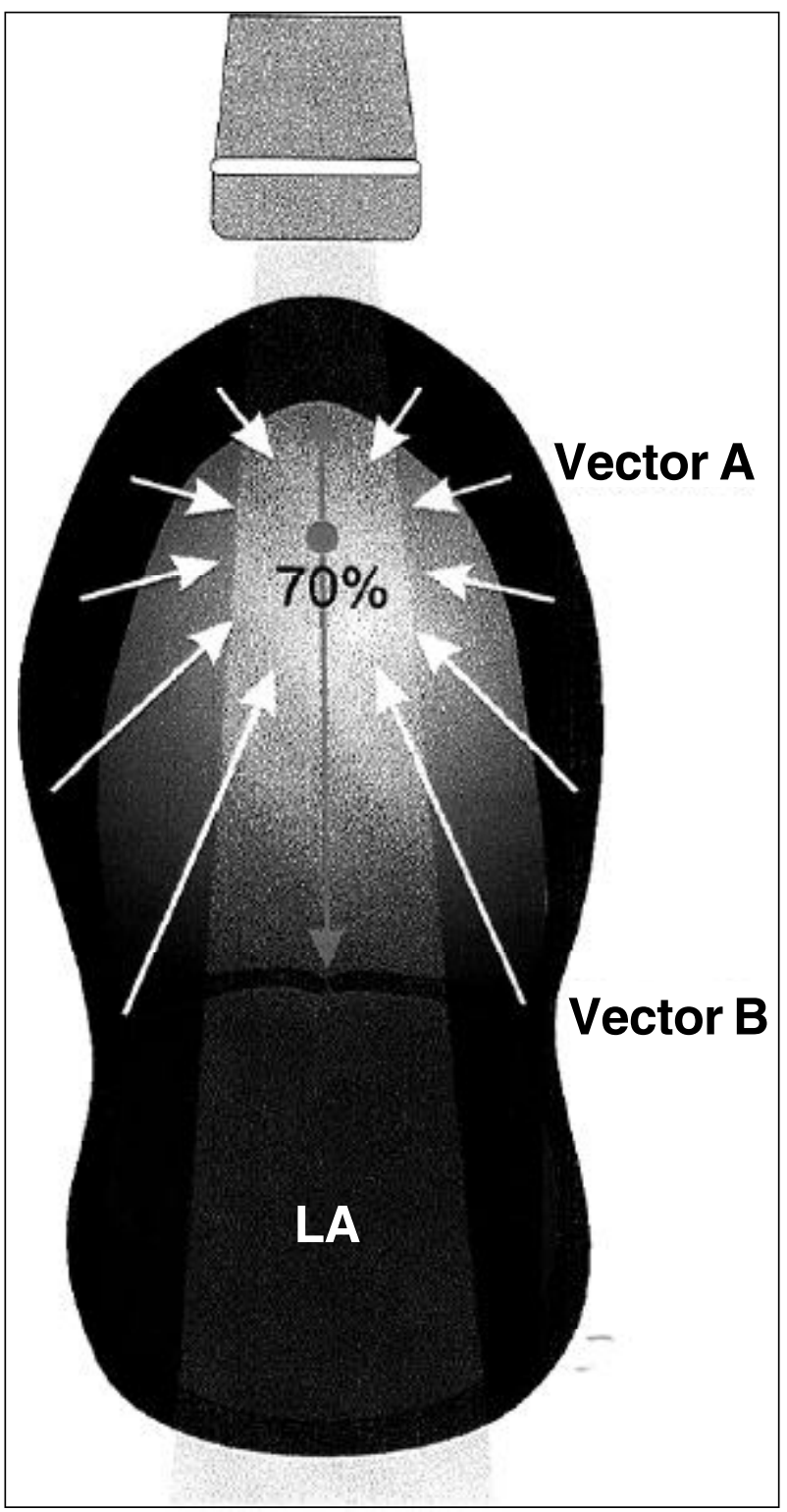

Fig. 5 - The resulting vector of the myocardial movement is directed by a point inside the ventricle at approximately $70 \%$ of its longitudinal diameter. With regards to the ultrasound beam, the B vector is located almost parallel while the A vector is almost perpendicular. This fact is justified partly by the smaller velocities found in the apical region, when compared with those in to the basal region, because the velocity detected by Doppler is inversely proportional to the consensus of the angle formed by the vector and the ultrasound beam. LA- left atrium. a certain segment, when comparing the 2 phases of the cardiac cycle.

If we assume that the area of a section of the ventricular bases, near the mitral annulus, is much greater than the area of a section of the apical region, it makes sense that the contraction and relaxation of the base is more rapid than that of the apex. Otherwise, it would be difficult for the ventricle to fill and empty itself, which would be a contradiction.

Our study also shows that although the diastolic phase is longer than the systolic and therefore, mean myocardial motion velocity in diastole is less than in systole, because the myocardium travels the same space in the 2 phases, the same thing does not occur with the maximum motion velocity which, in our measures, always occurred during early filling, corresponding to the E wave of mitral inflow.

This suggests that diastolic distention caused by rapid atrial contraction is particularly important in the analysis of global diastolic function and that the active chemical phenomena that causes the ventricular contraction is not able to develop motion velocities comparable to those observed by the filling of the chamber, at least in relaxation conditions and normal distensibility.

The time in which the peak contraction and relaxation velocities occur in relation to the $\mathrm{R}$ wave is valid only to the mean cardiac frequency observed in our study $(72.8 \pm 10.7$ $\mathrm{bpm}$ ) because the R-R interval varies, in inverse proportion, according to cardiac frequency.

In conclusion, Doppler tissue imaging is a technique that enables, with some facility, the access of important physiological information related to the contraction and relaxation velocity of the ventricular myocardium.

In healthy individuals, maximum left ventricular segment motion velocities decreased from the bases to the ventricular apex, with certain proportionality between contraction and relaxation. These velocities have values dependent on the angle of motion between the myocardial wall and the ultrasound beam. The knowledge of these variables may be useful in the study of diseases that compromise myocardial contractility regionally or diffusely.

\section{References}

1. Satomura S. A study on examining the heart with ultrasonics. I Principles; II Instrument. Jpn Circ J 1956; 20: 227.

2. Picano E, Lattanzi F, Orlandini A, Marini C, L'Abbate A. Stress echocardiography and the human factor: the importance of being expert. J Am Coll Cardiol 1991; 17: 666-9.

3. McDicken WN, Sutherland GR, Moran CN, Gordon LN. Color Doppler velocity imaging of the myocardium. Ultrasound Med Biol 1992; 18: 651-4.

4. Isaaz K, Thompson A, Ethevenot G, et al. Doppler Echocardiography measurement of low velocity motion of the left ventricular posterior wall. Am J Cardiol 1989; 64: 66-75.

5. Sutherland GR, Stewart MJ, Groundstroen KWE, et al. Color Doppler myocardial imaging: a new technique for the assessment of myocardial function. J Am Soc Echocardiogr 1994; 7: 441-58.
6. Fleming D, McDicken WN, Sutherland GR, et al. Assessment of color Doppler tissue imaging using yest phantoms. Ultrasound Med Biol 1994; 20: 937-51.

7. Rychik J, Tian ZY. Quantitative assessment of myocardial tissue velocities in normal children with Doppler tissue imaging. Am J Cardiol 1996; 77: 1254-7.

8. Sutherland GR, Bijnens B, McDicken WN. Tissue Doppler echocardiography: historical perspective and technological considerations. Echocardiography 1999; 16: 445-53.

9. Palka P, Lange A, Sutherland GR, et al. Doppler tissue imaging: myocardial wall motion velocities in normal subjects. J Am Soc Ecocardiogr 1995; 8: 659-68.

10. Garcia-Fernandez MA, Moreno M, Coleman P, et al. Regional left ventricular diastolic dysfunction evaluated by tissue Doppler imaging as na earlier signal of myocardial ischemia. Eur Heart J 1995; 16: 129, 788. 
11. Rychik J, Tian Z. Quantitative assessment of myocardial tissue velocities in normal children with Doppler tissue imaging. Am J Cardiol 1996: 77; 1254-7.

12. Galiuto L, Ignone G, DeMaria A. Contraction and relaxation velocities of the normal left ventricle using pulsed-wave tissue Doppler echocardiography. Am J Cardiol 1998: 81; 609-14.
13. Garcia-Fernandez MA, Azevedo J, Puerta P, et al. Regional left ventricular diastolic dysfunction evaluated by Doppler tissue imaging as an earlier signal of myocardial ischemia: experimental study of induced ischemia and reperfusion in pigs. J Am Coll Cardiol 1996; 27: 299A.

14. Garcia MJ, Thomas JD. Tissue Doppler to assess diastolic left ventricular function. Echocardiography 1999; 16: 501-8. 